\title{
Thermography in laser powder bed fusion of metals: time over threshold as feasible feature in thermographic data
}

\author{
by S.J. Altenburg*, N. Scheuschner*, C. Maierhofer*, G. Mohr ${ }^{*, * *}$ and K. Hilgenberg*,** \\ * Bundesanstalt für Materialforschung und -prüfung (BAM), Unter den Eichen 87, 12205 Berlin, Germany, \\ simon.altenburg@bam.de \\ **Technische Universität Berlin, Institute of Machine Tools and Factory Management, Chair of processes and \\ technologies for highly loaded welds, Straße des 17. Juni 135, 10623 Berlin, Germany
}

\begin{abstract}
Thermography is one on the most promising techniques for in-situ monitoring of metal additive manufacturing processes. Especially in laser powder bed fusion processes, the high process dynamics and the strong focus of the laser beam cause a very complex thermal history within the produced specimens, such as multiple heating cycles within single layer expositions. This complicates data interpretation, e.g., in terms of cooling rates. A quantity that is easily calculated is the time a specific area of the specimen is at a temperature above a chosen threshold value (TOT). Here, we discuss variations occurring in time-over-threshold-maps during manufacturing of an almost defect free cuboid specimen.
\end{abstract}

\section{Introduction}

By allowing economic on demand manufacturing of highly customized and complex workpieces, metal based additive manufacturing (AM) has the prospect to revolutionize many industrial areas. A fundamental requirement for AM to become applicable in most fields is the ability to guarantee the adherence to strict quality and safety standards [1]. A possible solution for this problem lies in the deployment of various in-situ monitoring techniques [2]. For most of these techniques, the application to AM is not yet developed to a sufficient extent. To help overcome this issue, we initiated the project "Process Monitoring of AM" (ProMoAM). In this project, a wide range of in-situ process monitoring techniques, including active and passive thermography, optical tomography, optical emission spectroscopy, particle emission spectrometry, eddy current testing and X-ray methods are applied to laser metal deposition, laser powder bed fusion (L-PBF) and wire arc AM. In successive steps, the data acquired by these methods is fused and compared to the results of reference methods such as computed tomography $(\mathrm{CT})$ and ultrasonic immersion testing. The goal is to find reliable methods to detect the formation of defects during the building process.

In this work, we focus on thermography, one of the most promising techniques for in-situ monitoring of the L-PBF process [3]. Due to the high process dynamics and the strong focus of the laser beam, the thermal history of the produced parts is very complex. If also the high temperature range (above and close to the melting temperature of the material) is to be analysed, thermography has to be performed with high temporal and spatial resolution. The resulting large amount of acquired data has to be condensed to useful information, i.e., suitable features for part quality assessment. Further steps of data fusion have to be performed, in the ideal case online and layer-wise or even already during the exposition of a layer. The time a specific area of the specimen is at a temperature above a chosen threshold value (time over threshold TOT) is a sensitive quantity to condense process dynamics into an easy to calculate feature $[3,4]$. Here, we show that during the manufacturing of a simple defect free cuboid specimen significant variations in the TOT can be observed and explained, both within single layers and with increasing build height. The results presented here are based on measurement results of a previous study [4] and give a more detailed analysis of the feature TOT than presented in the original publication.

\section{Experimental setup and specimen}

A detailed description of the experimental setup and specimen design is given elsewhere [4]. We used AISI 316L austenitic stainless steel powder on a commercial L-PBF system SLM280 HL (SLM Solutions Group AG, Germany), equipped with a single $400 \mathrm{~W}$ continuous wave $(\mathrm{cW})$ ytterbium fibre laser emitting at $1070 \mathrm{~nm}$. A layer thickness of $50 \mu \mathrm{m}$ was chosen for the process, using a hatch distance of $120 \mu \mathrm{m}$, a laser power of $275 \mathrm{~W}$, a laser spot diameter of $80 \mu \mathrm{m}$ and a scan velocity of $700 \mathrm{~mm} / \mathrm{s}$. For the in-situ monitoring of the L-PBF process regarded here, a mid-wave infrared (MWIR) camera sensitive in the spectral range from $2 \mu \mathrm{m}$ to $5.7 \mu \mathrm{m}$ was chosen (Infratec ImagelR8300, InfraTech $\mathrm{GmbH}$, Germany) and operated at a sampling frequency of $900 \mathrm{~Hz}$. The camera is calibrated for black body radiation in a temperature range between $623 \mathrm{~K}$ and $973 \mathrm{~K}$, which corresponds to an integration time of $90 \mu \mathrm{s}$. The camera was placed outside of the build chamber on top of the L-PBF system and observed the process through a sapphire window in the ceiling of the build chamber. The use of an objective with $100 \mathrm{~mm}$ focal length resulted in an optical resolution of $100 \mu \mathrm{m} / \mathrm{pixel}$ in the thermogram. As a specimen, we regard only the lower part of a specimen built for another study [4], a cuboid with a $10 \mathrm{~mm} \times 10 \mathrm{~mm}$ base and $5 \mathrm{~mm}$ height. Reference CT scans with a voxel size of $7.12 \mu \mathrm{m}$ showed only a low 
number of single pores in the specimen; thus, it can be regarded as virtually defect free. The scan strategy was set to alternating horizontal and vertical bidirectional line-scanning from top to bottom and left to right in the thermographic images, respectively.

\section{Results and discussion}

Since the MWIR camera was calibrated for black body radiation, the temperatures that were acquired in this work were only apparent temperatures (ATs) and far below the thermodynamic temperatures in the process. Several influences such as the low emissivity of stainless steel, non-unity transmission of the optical elements in the optical path and high spacial temperature gradients on the investigated surface contribute to this reduction of the acquired temperature. To emphasize this, we give the AT in apparent Kelvin, $\mathrm{K}_{\mathrm{a}}$. We extended our analysis window for the ATs down to $550 \mathrm{~K}_{\mathrm{a}}$, interpolating the manufacturers calibration outside the specified temperature interval $\left(623 \mathrm{Ka}_{\mathrm{a}}\right.$ to $\left.973 \mathrm{Ka}\right)$.

Fig. 1 gives an overview of typical thermographic data. Fig. 1 a shows a single thermogram of the process, illustrating that there was a certain amount of spatter ejected from the molten pool traveling through the image, which might complicate certain image processing steps. The chosen acquisition frequency of $900 \mathrm{~Hz}$ at a spacial resolution of $100 \mu \mathrm{m} / \mathrm{pixel}$ and a scan velocity of $700 \mathrm{~mm} / \mathrm{s}$ resulted in a movement of the melt pool of approximately 8 pixels between consecutive frames, which is illustrated in Fig. $1 \mathrm{~b}$ to $\mathrm{d}$. Thus, especially in the analysis of high temperature features in the dataset, aliasing effects occur due to this subsampling (compare Fig. 2 c).

Fig. 1 e shows the time development of the AT at three different single pixel positions in the specimen, as depicted in Fig. $1 \mathrm{a}$ and Fig. 2 . The positions were chosen in such a way that the melt pool reached its maximum temperature at the specific position while passing, thus there is no influence by the described subsampling effect regarding maximum temperatures here. As expected for the chosen build parameters, the AT rose multiple times, thus each volume element was reheated several times during each layer exposition. There are distinct differences in the AT evolutions, depending on the position in the sample: The blue curve corresponds to a position within the first hatch in this layer. Since there is no preheating due to close by hatches that were heated by the laser beforehand, the temperature rise is sudden, and the reached maximum temperature is below the value of the other curves. The red curve corresponds to a position in the middle of the sample and a preheating can be observed at negative times. The cooling down is similar to the case of the position at the upper rim of the specimen. The green curve corresponds to a position at the last scan vector of the specimen. Thus, the preheating is similar to the middle case (red curve), but the cooling behaviour differs. At temperatures above approximately $700 \mathrm{~K}_{\mathrm{a}}$ the cooling is slower than in the other cases, which can be best observed between the maximum temperature at $0 \mathrm{~ms}$ and the first reheating by the returning laser spot, reaching a new local maximum at approximately $20 \mathrm{~ms}$. This effect can be explained by the missing cooler solidified material at the lower hand side in the image, since for the last scan vector the parts ends here and there is only powder with much lower thermal conductivity at the rim of the specimen. This explanation is supported by the missing of this discrepancy in the first layers of the build, as explained below (compare also Fig. 4). On the other hand, the missing reheating by further scan vectors in the lower end of the part leads to an increased cooling rate at later times (and lower ATs), which can be observed for times later that approximately $50 \mathrm{~ms}$.

The grey curve in Fig. $1 \mathrm{f}$ shows the time evolution of a single pixel marked by the grey circle in Fig. 1 a and illustrates two effects: First, since the position is not chosen to match a local maximum temperature during the passing of the laser, the shift along the time axis to set the maximum AT to $0 \mathrm{~s}$ does not result in a well-defined joint time axis for comparison to, e.g., the red curve. This illustrates complications in analysing the time evolution of the AT directly instead of the TOT. Secondly and not obviously, the cooling behaviour above $700 \mathrm{Ka}$, especially between $50 \mathrm{~ms}$ and $100 \mathrm{~ms}$, is quite slow and rather comparable to the green line in Fig. 1 e, possibly indicating the presence of a defect. Actually, this position for analysis of the time evolution of the AT was selected by a signature in the TOT map in Fig. 2 b (see below).
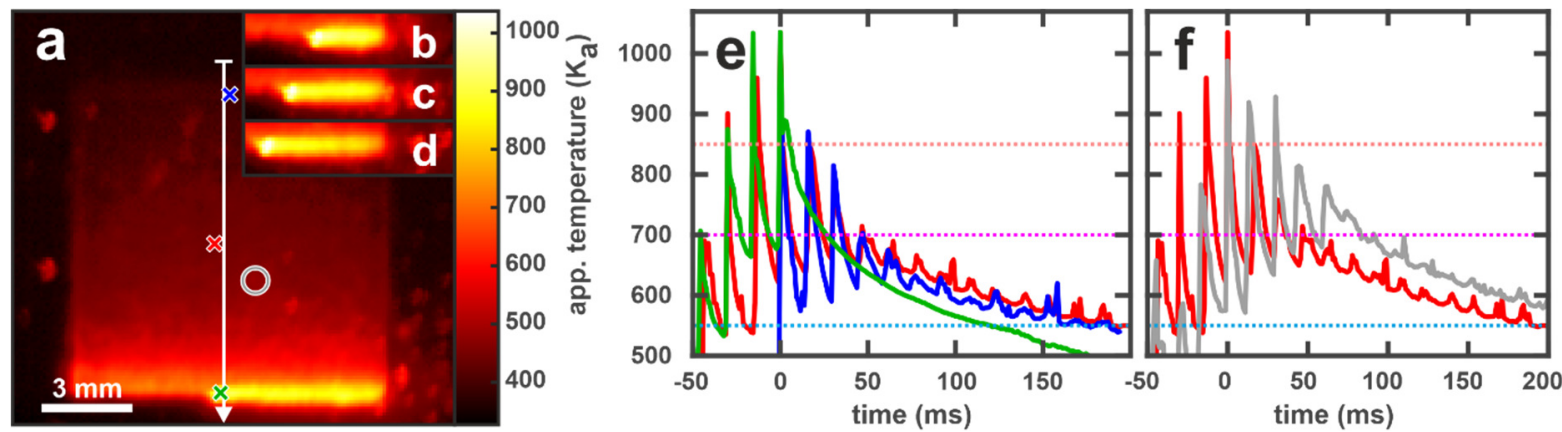

Fig. 1: a: Thermogram of layer 43 of 100 consisting of $192 \times 176$ pixels. In this layer, as in all odd layers, the exposition was performed in horizontal hatches from top to bottom in the image. In the shown frame, the laser moves from right to left in the last vector of the layer. Crosses denote positions of time evolutions shown in e, the grey circle indicates the position of the time development in $\mathrm{f} . \mathrm{b} / \mathrm{c} / \mathrm{d}$ : Sections of three consecutive frames. The maximum in the thermogram moves by approximately 8 pixels from frame to frame. e: Time evolution of the AT in the positions (single pixels) marked by crosses in a. The time axes were shifted for each position to match the maximum at 0 s. The threshold 
values of the TOT-analysis are indicated by dashed lines. $f$ : Time evolution of the AT in the positions (single pixels) marked by the red cross and the grey circle in a (see also Fig. 2).

These observations in the time development of the ATs at single points need a certain amount of data preprocessing (shift along the time axis for matching maximum temperature) and may even be less obvious and more complicated to quantify automatically, when analysing positions closer to the left or right turning points of the laser (and the specimen), since additional asymmetries in the time evolution of the AT occur. The same is true when analysing positions between maximum positions of the melt pool, since the time of maximum AT becomes less well defined in this case (compare Fig. $1 \mathrm{f}$ ). However, when the TOT is analysed instead, the data analysis is very fast and simple while the effects described above can still be observed in the data. This is discussed in the following.

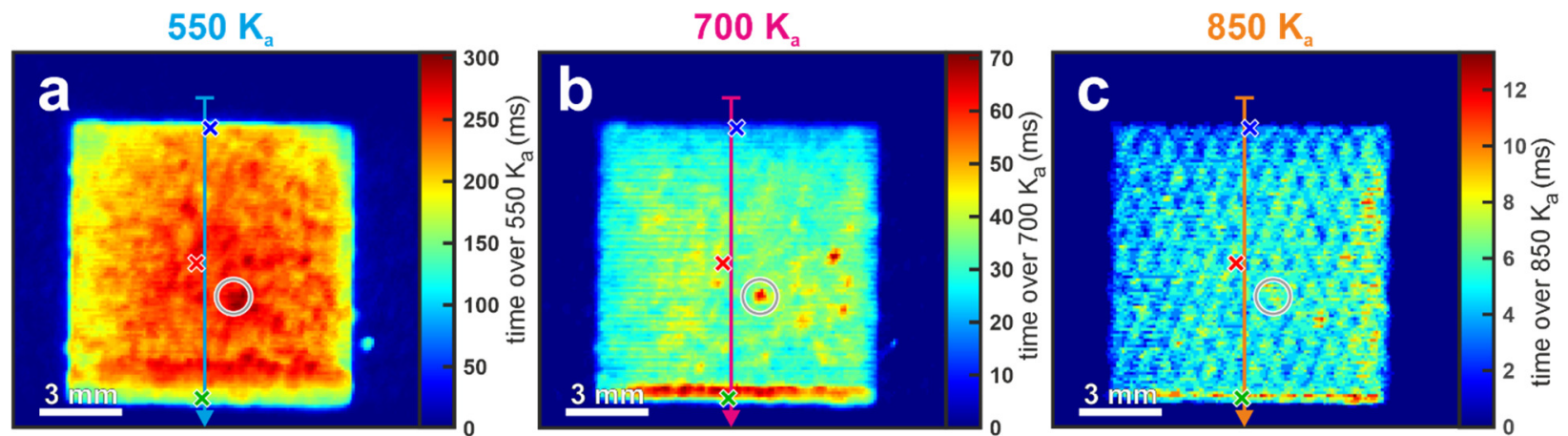

Fig. 2: TOT maps of layer 43 (see Fig. 1) for different threshold values. The colour scale is given in ms. Profile positions for Fig. 3 are indicated by lines, positions of time evolutions of Fig. 1 e (f) are denoted by crosses (a circle). a: Threshold value $550 \mathrm{Ka}$. b: Threshold value $700 \mathrm{Ka}$. c: Threshold value $850 \mathrm{Ka}$. A regular pattern caused by temporal subsampling can be observed (compare Fig. 1, b to d).

Figure 2 shows TOT maps at three different threshold values of $550 \mathrm{Ka}, 700 \mathrm{Ka}$ and $850 \mathrm{Ka}$. These values have been selected because for a threshold value of $550 \mathrm{Ka}$, the cooling down time factors at lower temperatures are considered, for $700 \mathrm{Ka}$, the cooling down time factors at higher temperatures are considered, but without any influences of subsampling, and for $850 \mathrm{Ka}$, the influence of subsampling is shown. These threshold values are indicated by the dashed lines in Fig. 1 $\mathrm{e}$ and $\mathrm{f}$. The described strong subsampling effect is clearly visible in Fig. $2 \mathrm{c}$ at a threshold value of $850 \mathrm{Ka}$ and renders these data to be very complicated for further analysis. However, in Fig. $2 \mathrm{~b}\left(700 \mathrm{~K}_{\mathrm{a}}\right)$ the subsampling effect is not present. The decreased cooling rate at temperatures above $700 \mathrm{~K}_{\mathrm{a}}$ that was observed in Fig. $1 \mathrm{e}$ is clearly observable at the lower end of the specimen by an increased TOT. There are additional spots of increased TOT within the specimen that are also caused by a locally decreased cooling rate, as shown in Fig. $1 \mathrm{f}$ for the spot indicated by the grey circle in Fig. $2 \mathrm{~b}$. This feature might be an indication for a defect at the current layer or at one of the previous layers, that disturbs the heat flow. However, the reheating of the material during the manufacturing of subsequent layers influences the material and might heal a possible defect. Correlations of TOT deviations with reference CT measurements were presented in a previous study [4].

Fig. 2 a shows the TOT map for a threshold value of $550 \mathrm{Ka}$. The increase in the TOT at the lower rim of the specimen that is visible in the TOT $(700 \mathrm{Ka})$ is replaced by a relative decrease, as explained above. Additionally, the TOT $\left(550 \mathrm{~K}_{\mathrm{a}}\right)$ is decreased in the upper corners of the specimen, probably also caused by a lack of heating in the surrounding area (only one quadrant is heated compared to 2 quadrants at the rims). At the position of the probable defect (grey circle), there is also a slightly increased value of the TOT $(550 \mathrm{Ka})$.
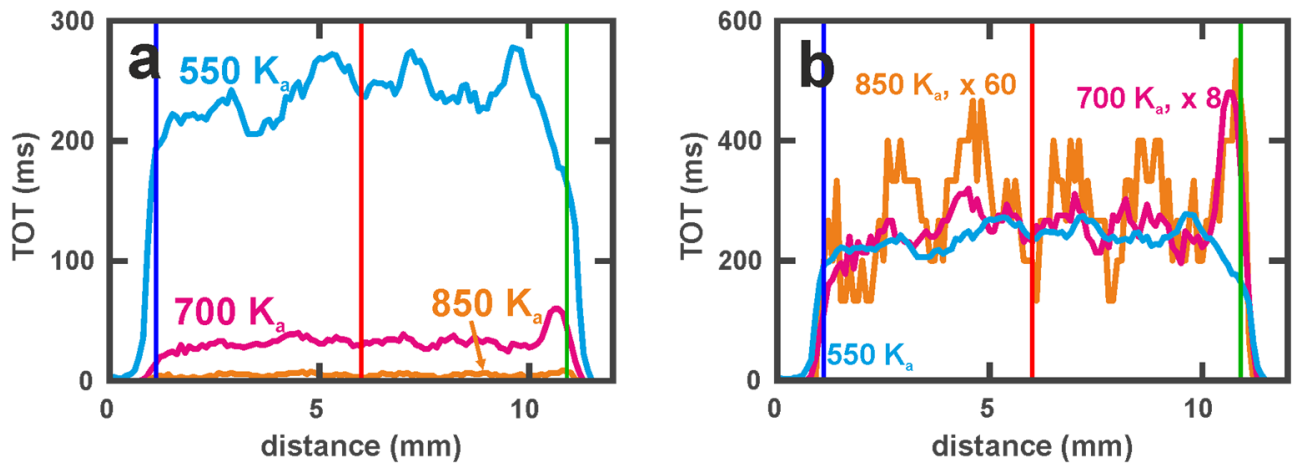

Fig. 3: Profiles through the TOT-maps of Fig. 2. Positions of the time evolution plots in Fig. 1 e are indicated by vertical lines of the corresponding colour. a: to scale. $b$ : adjusted TOT values for relative comparison, multiplication of the $700 \mathrm{~K}_{a}$ and $850 K_{a}$ data as indicated. 
Fig. 3 shows profiles through the TOT maps of Fig. 2 to further illustrate the effect of the increased $\left(700 \mathrm{Ka}_{\mathrm{a}}\right.$, $\left.850 \mathrm{~K}_{\mathrm{a}}\right)$ or decreased $\left(550 \mathrm{~K}_{\mathrm{a}}\right)$ TOT values at the rims of the specimen. In Fig. $3 \mathrm{~b}$ the time scales have been adjusted to each other for enabling a better comparison of the contribution of different temperature intervals to the temporal behaviour of the cooling down curves.
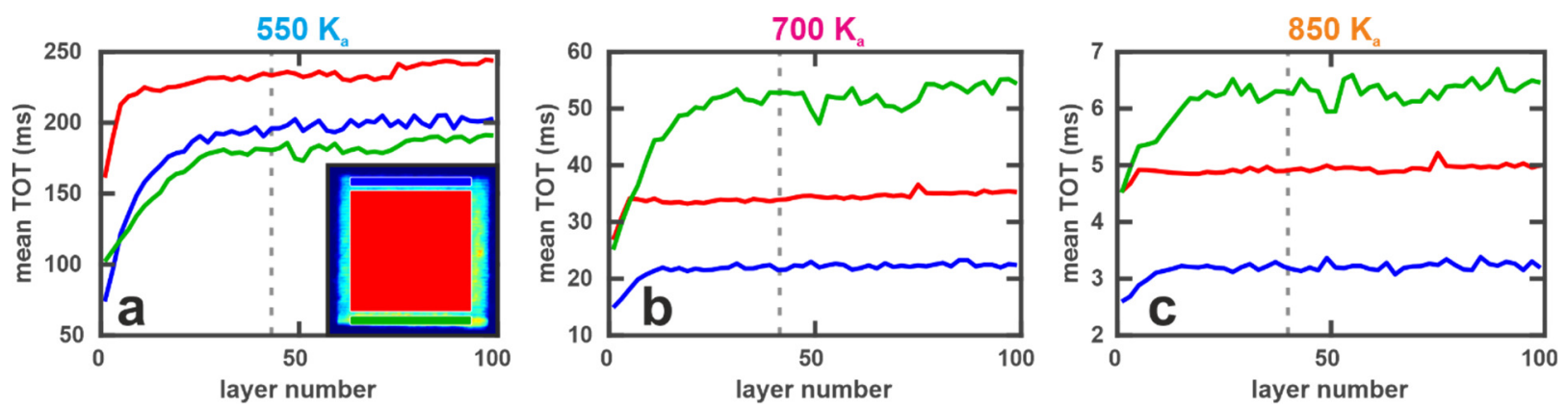

Fig. 4: Evolution of the TOT with increasing build height (increasing layer number) for the different threshold values, averaged in the areas indicated in the inset of a, representing the corresponding time evolutions of Fig. 1 e. Due to the rotation of the scan vector between even and odd layer numbers, only odd layer numbers with horizontal scanning are analysed. a: Threshold value $550 \mathrm{Ka}$. b: Threshold value $700 \mathrm{Ka}$. c: Threshold value $850 \mathrm{Ka}$. The grey dotted line indicates layer 43, which was analysed in detail in Fig. 1-3.

To further analyse the influence of the build height on the TOT and the observed geometry induced features, Fig. 4 shows average values of the TOT for the upper, the middle and the lower part of the specimen for the three different threshold values. For all three threshold values and all regions, the TOT increases strongly with the first layers and then slightly further up to the end of the observation interval at layer 100 . For Fig. 4 a (TOT $\left(550 \mathrm{~K}_{\mathrm{a}}\right)$ ), the differences between upper and lower rim are minute and the dynamic of the scan strategy appears to play only an insignificant role. The TOT is reduced at the rim compared to the centre of the specimen, since lateral heat flow to the surrounding is increased here. The TOT is reduced for the first layers, since the low part height and low accumulated heat in the build plate lead to a more effective heat transport to the base plate than in higher layers. For the higher threshold values $700 \mathrm{~K}_{\mathrm{a}}(\mathrm{Fig} .4 \mathrm{~b})$ and $850 \mathrm{Ka}$ (Fig. 4 c), the dynamic of the scan strategy is important. At the upper rim of the specimen (blue curve), the TOT is always lower than in the rest of the specimen since there is no preheating by a scan of neighbouring areas. At the lower rim of the specimen that is scanned last (green) the TOT is increased due to missing heat flow to adjacent solid material, as explained above. This interpretation is supported by the missing difference to the centre region (red curve) for the first layers. In the first layers, the heat flows directly into the base plate and the thermal barrier by the powder builds up with increasing part height. The slight increase of the TOT over the whole build process is in accordance with the observation of decreasing heat transfer through the growing part during manufacturing of parts in L-PBF [6].

\section{Summary and Outlook}

In summary, we monitored an L-PBF manufacturing process of a cuboid specimen by thermography and analysed the obtained data by calculating the time each part of the specimen was above a certain apparent threshold temperature. Depending on the threshold value, the part geometry and the scanning strategy influence the TOT-feature. In order to use this easily and fast computable feature for possible defect detection, these influences have to be taken into account to avoid false positive defect detection. Simplified numerical simulations based on part geometry and scan strategy could help to predict TOT variations in the future.

Although very promising results in the analysis of TOT thermography data of an almost defect free specimen production have been found, further studies are needed, especially regarding the formation of defects. Different kinds of defects or their formation mechanisms might influence the TOT in different ways. For example, the ejection of loosely bounded material that cools down relatively slowly leads to a locally strongly increased TOT directly in the layer of occurrence. In the subsequent layer, however, a small increase in TOT at this location gets possibly registered, due to heat accumulation above the defect. Contrarily, not completely molten powder leading to lack-of-fusion pores might cause an increase of the TOT in the subsequent layer and not in the layer of origin. Thus, for a thorough understanding of the process and its influence on the TOT, extensive studies are needed.

This research was funded by BAM within the focus area Materials.

\section{References}

[1] Tapia G., Elway A., A review on process monitoring and control in metal-based additive manufacturing. Journal of Manufacturing Science and Engineering, 136.6 (2014), 060801-1/10

[2] Grasso M., Colosimo B. M., Process defects and in situ monitoring methods in metal powder bed fusion: a review. Measurement Science and Technology 28.4 (2017): 044005, 25 pp.

[3] Hooper P. A., Melt pool temperature and cooling rates in laser powder bed fusion, Additive Manufacturing, 22 (2018), pp. 548-559, doi: https://doi.org/10.1016/j.addma.2018.05.032. 
[4] Mohr G., Altenburg S. J., Ulbricht A., Heinrich P., Baum D., Maierhofer C., Hilgenberg K., In-situ defect detection in laser powder bed fusion by using thermography and optical tomography-comparison to computed tomography, Metals 10.1 (2020) https://doi.org/10.3390/met10010103

[5] Lough C. S., Wang X., Smith C. C., Adeniji O., Landers R. G., Bristow D. A., Kinzel E. C., Use of swir imaging to monitor layer-to-layer part quality during slm of 304I stainless steel, Proceedings of the 29th Annual International Solid Freeform Fabrication Symposium, Austin, TX, USA, 2018. p. 13-15.

[6] Mohr G., Altenburg S. J., Hilgenberg K., Effects of inter layer time and build height on resulting properties of 316I stainless steel processed by laser powder bed fusion, Additive Manufacturing - Vol. 32p. 101080, 2020. https://doi.org/10.1016/j.addma.2020.101080 